postulate that the flow of heat could be treated as that of an indestructable substance. In view of its antecedence in the caloric theory, and of its subsequent universal acceptance, it is hard to appreciate that Fourier had great difficulty in persuading Laplace, Biot and Poisson of its validity. Their doubts about his physics were reinforced by parallel doubts about his non-rigorous treatment of trigonometrical series, but in both instances his instincts proved correct. Refusing to be discouraged by the objections, Fourier developed his treatment of the heat conduction problem into one of the classical triumphs of physics, and incidentally developed his use of trigonometrical series in the solution of his differential equations of conduction. All that is well brought out by Dr Herivel, who has deliberately concentrated on Fourier as a physicist and a man, on the grounds that Fourier the mathematician has been well covered. But I would have been grateful had some of Fourier's mathematics been included in the same volume because although, even if we agree with Dr Herivel that "It would be unrealistic to strike a balance between Fourier the man and Fourier the savant", the interplay of mathematics

\section{Tissue connection}

\section{Connective Tissue: Macromolecular Structure and Evolution. (Molecular Biology-Biochemistry and Biophysics, vol. 19.) By Martin B. Mathews. Pp. xii +318 . (Springer: Berlin and New York; Chapman and Hall: London; May 1975.) $£ 15.30$.}

THIS monograph, the 19 th in the series Molecular Biology-Biochemistry and Biophysics, is an excellent review of the macromolecular structure and evolution of connective tissue. During recent years the advances in this field have been substantial indeed and this is the first time that an attempt has been made to consider this vast, widely scattered literature in the context of evolution and taxonomy.

The general construction and arrangement of the book are admirable. The first six chapters, amounting to about half the text, consist of a concise survey of the biochemistry of the macromolecular constituents of connective tissue: collagen, elastin, structural glycoproteins and polyanionic proteoglycans. The emphasis lies very much on the description of primary levels of organisaton, although relevant conformational data are mentioned and occasionally described. Each chapter also presents a most comprehensive account of the comparative biochemistry of these macromolecules, leading to considerations of molecular phylogeny. These considerations are, and physics in Fourier's achievements must be a phenomenon of great interest. But, even regarding Fourier as a physicist alone, it is hard to understand why there is no reference in the book to Fourier's invention of the method of dimensions, which almost every subsequent physicist has used (Maxwell, for example) and which is a sure criterion of Fourier's insight.

Dr Herivel almost conveys a sense of disappointment that conspicuous though Fourier's civic role was it "can merit no more than a footnote in the history of the period whereas both as a physicist and as a mathematician he was undoubtedly one of the major figures of the nineteenth century and beyond". But what is really remarkable, as in the case of Handel, is that interspersed among the passages of a tempestuous and worldly career, Fourier should have achieved such heights of contemplatiy creation. Men of science who still have to battle with administrators who are reluctant to regard them as equals may point with gratitude to the example of Fourier. And their gratitude may be extended to Dr Herivell for this, within its limits, excellently annotated and documented account of Fourier and his times.

however, hampered by the rather limited amount of structural information, particularly concerning amino acid sequences, which is now available. The coverage of collagen and glycosaminoglycans is extensive and this makes even more noticeable the somewhat limited space given to the discussion of elastin. This is unfortunate as the structure of that protein must still be regarded as controversial and the reader would have been helped by a more critical appraisal of the literature.

Professor Mathews' own particular interests-changes in cartilage biochemistry with embryonic development, maturation and ageing, and the taxonomic distribution of glycosaminoglycans-are presented in the next three chapters. These contain separate sections for individual tissues, among which particular emphasis is given to cartilage, bone and notochord. Apart from their evolutionary significance, they are a mine of information and contain some original, unpublished work from the author's laboratory.

The last chapter is concerned mainly with the molecular properties and interactions of collagen and proteoglycans and because it encompasses so many facets of the subject, it should appeal to readers from many other disciplines.

The style is direct and unpretentious and the author admirably combines erudition and lucidity of exposition. This book will be most welcome to those working in this field.

\section{A. Serafini-Fracassini}

\section{Environmental}

\section{mechanics and}

\section{thermodynamics}

Fluid Mechanics and Thermodynamics of our Environment. By $\mathrm{S}$. Eskinazi. Pp. $x v+422$. (Academic : New York and London, February 1975.) $\$ 26.00 ; £ 12.50$.

IN comparison with other books on the motion of fluids by the same author, this one is a little disappointing in its production and layout. According to the preface the word 'environment' takes a variety of meanings and it is a word which is certainly made use of far too often in the text; usually, the more precise term 'atmosphere' or 'ocean' would suffice in its stead. The book is aimed at engineering students interested in air-borne and water-borne pollution but, though there is an obvious need for a concise introductory text which covers this field, this presentation leaves one with the impression that it is partly an attempt to jump on the current bandwagon of concern over man's use of the Earth as a waste disposal unit. The diagrams are the worst feature of the book and are sometimes amateurish in style. Their reproduction is usually on so small a scale that details tend to be obscured.

On the good side, the book is a useful introduction to both physical meteorology and occanography, necessary parts of the curriculum of anyone studying pollution. Usually, these two subjects are treated separately but here the author has brought together their simpler aspects in one unified text and has attempted to present the material in a manner not requiring formal courses in fluid mechanics and thermodynamics as prerequisites. On the whole he has succeeded although a good background in physics and mathematics is essential for a full understanding of the content of the book. Nevertheless, in one or two places, the reader is plunged into discussions of turbulent flows without the benefit of earlier treatment of simpler laminar models. Although it can be argued that flows in the atmosphere are rarely laminar, the difficulties of the treatment of the motions of a real atmosphere are often daunting to an undergraduate with no training in hydrodynamics.

The book should prove most useful to the experienced scientist or engincer who requires a knowledge of the rudiments of meteorology and oceanography. It may also find some application in courses in geophysics which are not solely confined to a study of the solid Earth.

C. W. Titman 